\title{
Impact of 6-Month Exercise Program on Weight Status and Associated Biomarkers in Mild Cognitive Impairment
}

\author{
Mary Beth Spitznagel, ${ }^{1,2}$ Lindsay A. Miller, ${ }^{1}$ Vanessa Potter, ${ }^{2}$ \\ Ellen Glickman, ${ }^{3}$ and John Gunstad ${ }^{1,2}$ \\ ${ }^{1}$ Department of Psychology, Kent State University, Kent, OH 44242, USA \\ ${ }^{2}$ Department of Psychiatry, Summa Health System, 75 Arch Street, Suite G2, Akron, OH 44304, USA \\ ${ }^{3}$ Department of Exercise Science, Kent State University, Kent, OH 44242, USA
}

Correspondence should be addressed to Mary Beth Spitznagel, mspitzna@kent.edu

Received 21 March 2011; Accepted 17 May 2011

Academic Editors: T. Kovács, C.-R. Lai, E. Mukaetova-Ladinska, and P. J. Twomey

Copyright (C) 2011 Mary Beth Spitznagel et al. This is an open access article distributed under the Creative Commons Attribution License, which permits unrestricted use, distribution, and reproduction in any medium, provided the original work is properly cited.

\begin{abstract}
Unintentional weight loss in Alzheimer's disease (AD) predicts morbidity and mortality, but effectiveness of dietary supplementation is reduced in those with low body mass index. Exercise may be beneficial, particularly if started early. Thirty-one participants with mild cognitive impairment (MCI) completed measures of cardiovascular fitness, eating behavior, cognitive function, and body composition before and following a 6-month exercise program. Ghrelin, leptin, and brain-derived neurotrophic factor were measured to elucidate the relationship between cognition and response to exercise. Significant baseline relationships emerged among appetite and both cardiovascular fitness and neurocognition. Following the exercise program, improvements in cardiovascular fitness and healthy eating habits occurred, body composition and biomarkers remained stable, and cognition declined. This study suggests exercise may benefit persons with MCI by promoting healthier eating habits and possibly stabilizing body composition and biomarkers. Although increased fitness did not stabilize cognitive decline in MCI, exercise may positively impact eating behavior.
\end{abstract}

\section{Introduction}

Unintentional weight loss is associated with Alzheimer's disease $(\mathrm{AD})[1]$ and often precedes onset of cognitive decline and diagnosis [2]. Significant weight loss (greater than $4 \%$ of body weight in 1 year) independently predicts morbidity and mortality in older adults [3]. Loss of lean mass has recently been linked to brain atrophy and decreased cognitive performance in $\mathrm{AD}$ [4]. In contrast, an attenuated loss of body mass in older adults is associated with neuroprotection [5].

The mechanisms linking weight loss and $\mathrm{AD}$ are not fully understood, but persons with early stage $\mathrm{AD}$ demonstrate decreased food intake and eating behavior disturbances [6]. Poor nutritional status and appetite loss are common in persons with $\mathrm{AD}$ [7], and recent research suggests a direct link between $\mathrm{AD}$ and dietary patterns [8]. High-energy nutritional supplements are often utilized with individuals at risk for weight loss; however, supplementation appears to offer reduced benefit for individuals with very low body mass [9], underscoring the need for other interventions.

One possible intervention targeting both weight loss and the cognitive decline of AD may be exercise. Physical activity may improve the sensitivity of the body's satiety system, in turn increasing appetite [10]. Although the body's initial response to exercise may be weight loss, food intake typically increases to compensate for expended energy [11]. In addition, physical fitness and activity are linked with better neurocognitive outcome in older adults, including better neuropsychological test performance and decreased pathology on neuroimaging [12-16]. Increased cardiorespiratory fitness is associated with reduced brain atrophy in $\mathrm{AD}$ [17], and a recent population-based study shows that moderate exercise in mid- and late life is associated with decreased risk of developing MCI [18]. No study has directly assessed prevalence of sedentary lifestyle in persons with $\mathrm{AD}$, though 
only $2.5 \%$ of healthy older adults achieve recommended activity levels [19]. This pattern of inactivity may exacerbate weight loss, whether through decreased appetite or loss of lean muscle mass.

In this context, the relationship between declining cognition and weight loss may be further elucidated by biomarkers associated with eating behavior. Ghrelin, leptin, and brain-derived neurotrophic factor (BDNF) all have demonstrated relationships with eating behavior, appetite, or weight maintenance [20-22]. These biomarkers may be positively impacted by exercise or weight loss; research links obesity to increased leptin [23], and exercise decreases ghrelin [24] while increasing BDNF [13]. However, these biomarkers not only appear to be related to eating and weight, but are also linked with cognition. Specifically, there is evidence that increased ghrelin [25] and leptin [26] and decreased BDNF [27] are associated with poorer cognitive performance in healthy older adults. BDNF, a protein important for neuronal development, maintenance, and function, is reduced in patients with $\mathrm{AD}$ and vascular dementia relative to healthy older adults $[28,29]$. In addition, higher serum BDNF is associated with better cognitive test performance in healthy older adults [26]. High levels of leptin, an adipokine hormone typically linked to eating behavior through its action in the hypothalamus and striatal regions, are associated with smaller volumes of some frontal structures [30]. Consistent with these structural findings, elevated leptin levels are associated with reduced cognitive task performance, specifically frontal systems cognitive function [26]. Finally, the orexigenic hormone, ghrelin, is associated with increased disinhibition [31] and negatively correlated with several cognitive domains, including verbal memory, working memory, and naming [25]. Each of these biomarkers also has a demonstrated association with inflammation [32-34], and inflammation, in turn, has been linked to dementia including $\mathrm{AD}[33]$.

Given these findings, it is possible that structured exercise may help to stabilize weight loss in persons at risk for $\mathrm{AD}$ and perhaps attenuate the risk of mortality or disability in this population; these biomarkers may further inform these relationships. To clarify this possibility, older adults with mild cognitive impairment (MCI) participated in a structured exercise program for 6 months. Measures of cardiovascular fitness, cognitive function, body composition, eating behavior, and circulating biomarkers ghrelin, leptin, and BDNF were completed at baseline and upon completion of the exercise program. We hypothesized that baseline eating behavior and body composition would be positively associated with cardiovascular fitness, cognition, and associated biomarkers. Further, we hypothesized that the structured exercise program would be associated with improvement or stability in cardiovascular fitness, cognitive function, body composition indices, eating behavior, and the circulating biomarkers.

\section{Method}

The following procedures were approved by the local Institutional Review Board and conform to the provisions of the
Table 1: Participant demographic and clinical characteristics.

\begin{tabular}{lc}
\hline Demographic characteristics & Mean (SD) \\
Age (years) & $83.71(3.59)$ \\
Education (years) & $15.70(3.34)$ \\
Female (\%) & 58.1 \\
Clinical characteristics & $\%$ \\
Hypertension & 41.9 \\
CABG & 9.7 \\
Myocardial infarction & 12.9 \\
Diabetes & 6.5 \\
\hline
\end{tabular}

CABG: Coronary artery bypass graft surgery.

Declaration of Helsinki. All participants provided written informed consent prior to study involvement. Participant anonymity was maintained throughout the research protocol.

2.1. Participants. Thirty-one English-speaking individuals residing in a structured living facility participated. All participants had a global Clinical Dementia Rating (CDR) [35] score of 0.5 (questionable dementia or Mild Cognitive Impairment) rated by an experienced neuropsychologist following a battery of standard clinical neuropsychological tests. The 0.5 CDR designation results in a broadly defined category of cognitive impairment, including both amnestic and nonamnestic cognitive profiles. Table 1 shows demographic and clinical characteristics of the sample. Participants were excluded from study if they (1) demonstrated a CDR rating above or below 0.5 , (2) could not participate in exercise sessions due to physical limitations, (3) were younger than 55 years of age, or (4) were unable to independently provide informed consent.

\subsection{Measures}

2.2.1. Cardiovascular Fitness. Cardiovascular endurance was assessed with the 2-Minute Step Test [36]. In this task, participants were asked to march in place for two minutes, bringing each knee up to a marked target on the wall set at the individual's own midpoint between their hip and knee. The number of times the right knee met this point was counted.

2.2.2. Eating Behavior. Two self-measures related to eating behavior were utilized: the Council on Nutrition Appetite Questionnaire (CNAQ) [37] and Starting the Conversation: Diet (STCD) [38]. The CNAQ is an 8-item self-report measure that utilizes a Likert-type scale that provides an estimate of day-to-day appetite levels. It is psychometrically validated for use with the geriatric population [39]. A lower total score on this measure indicates poorer appetite. The STCD is a 7item self-report measure that also utilizes a Likert-type scale to provide a quick estimate of how healthy an individual's eating habits are. This measure examines food intake habits including healthy food intake such as lean protein, fruits, and vegetables, as well as indicators of poor food health habits, such as consumption of fast food, sugary beverages, desserts, 
and high-fat snacks. A lower total score on this measure indicates healthier eating habits.

2.2.3. Physical Composition Indices. Two measures of physical composition were taken: body mass index (BMI) and waistto-hip ratio (WHR). BMI is used to assess weight relative to height and is calculated by dividing body weight in kilograms by height in meters squared $\left(\mathrm{kg} / \mathrm{m}^{2}\right)$. For the WHR measure, waist and hip measurements of the individual are measured, and waist measurement is divided by that of the hip. For the measurement of the waist, the participant stands with both hands at his or her sides, feet together, with relaxed abdominals; horizontal measure is then taken at the narrowest part of the torso above the umbilicus and below the xiphoid process. For the measurement of the hips, the participant stands erect with feet together; horizontal measure is taken at the widest part of the buttocks. All measurements were taken in centimeters, on the skin surface, without compression of adipose tissue. All measures were completed in duplicate and the average utilized as the final measure.

2.2.4. Blood Markers. Biomarkers associated with exercise (i.e., the orexigenic hormone-ghrelin [24]; brain-derived neurotrophic factor (BDNF) - a protein important for neuronal function [13]), eating behavior (i.e., ghrelin) [20], obesity and appetite (i.e., the adipokine hormone-leptin) [21, 23 ] were conducted by collecting 12 -hour fasting venipuncture blood draws. All specimens were stored at $-80^{\circ} \mathrm{C}$ until assayed. Serum leptin levels were determined using the R\&D Systems Quantikine Human Leptin ELISA kit (R\&D Systems, Minneapolis, MN), which sandwiches human leptin between two monoclonal antibodies reacting against different epitopes on the leptin molecule. Serum ghrelin levels were determined using the LINCO Research Human Ghrelin (active) ELISA kit, a quantitative procedure with ghrelin sensitivity of $8 \mathrm{pg} / \mathrm{mL}$. Acidified specimens (final concentration of $0.05 \mathrm{~N} \mathrm{HCL}$ ) were tested. Serum BDNF levels were determined using the ChemiKine BDNF ELISA assay kit (Chemicon International, Temecula, CA), which sandwiches BDNF between a rabbit polyclonal antihuman BDNF antibody and a biotin labeled mouse monoclonal anti-human BDNF antibody. For all blood marker analyses, manufacturer's directions were followed, and specimens were tested in duplicate.

2.2.5. Neurocognitive Screening. Neurocognitive screening was conducted using the Modified Minimental State Exam (3MS) [40], an extended form of the MMSE which includes verbal fluency, abstract reasoning, and delayed word recall tasks. It has a maximum total score of 100 points. This measure is well validated and has excellent test-retest reliability $(r=.93)$ and good sensitivity (88\%) and specificity (90\%) [41].

2.3. Procedure. Prior to beginning and upon completion of the exercise program, participants completed neurocognitive screening, cardiovascular fitness measure, physical measures, 12-hour fasting blood draw, and self-report questionnaires. Participants took part in structured exercise sessions twice per week. Each exercise session was 60 minutes in length and included warm-up (15 minutes), aerobic exercise (15 minutes), resistance training (10 minutes), balance (5 minutes), and flexibility/cooldown (15 minutes). Different levels of each activity were demonstrated so that participants were able to begin at a level of personal comfort. Over the course of the intervention they were encouraged to progress to the next level as tolerated. All exercise sessions were supervised by a certified exercise physiologist and conducted over a 6month period.

2.4. Analyses. Pearson and bivariate correlations were first employed to examine relationships between appetite, eating patterns, BMI, and all other variables at baseline. Repeated measures ANOVA examined baseline to posttest changes in neurocognition and cardiovascular fitness. Repeated measures MANOVA was utilized to analyze measures within physical, self-report food intake questionnaires, and blood marker domains. Missing data was excluded pairwise.

\section{Results}

3.1. Relationships among Variables. A series of Pearson bivariate correlations determined the association among variables. Analyses showed that poorer reported appetite was associated with greater cognitive impairment (3MS; $r=0.53$, $P<0.01$ ) and reduced cardiovascular fitness (2-minute step test; $r=0.40, P<0.05)$. In addition, a significant positive relationship between BMI and leptin $(r=0.59, P<0.01)$ was observed. No significant relationships between eating patterns and other variables emerged.

3.2. Comparison of Baseline and Post-6-Month Structured Exercise Program. See Table 2 for descriptive statistics.

3.2.1. Physical Fitness. Repeated measures ANOVA from baseline to followup demonstrated improvement in cardiovascular fitness, as measured by the two-minute step test $(\lambda=.77, \mathrm{~F}(1,26)=7.86, P=0.009)$.

3.2.2. Self-Report Eating Behavior, Physical Composition Indices, and Biomarkers. Repeated measures MANOVA examining self-report eating behavior (CNAQ and STCD) was significant $[\lambda=.75, \mathrm{~F}(2,27)=4.61, P=0.02]$. Followup ANOVAs revealed that eating habits became healthier $[\mathrm{F}(1,28)=9.51, P=0.005]$, while no change in appetite was observed $[\mathrm{F}(1,28)=0.22, P=0.64]$. Repeated measures MANOVA of physical composition (BMI and WHR) showed that these indices remained stable $[\lambda=.95, \mathrm{~F}(2,28)=0.71$, $P=0.50]$. Repeated measures MANOVA of BDNF, leptin, and ghrelin showed no change in these biomarkers following the structured exercise intervention $[\lambda=.87, \mathrm{~F}(3,27)=1.40$, $P=0.26]$.

3.2.3. Neurocognition. A repeated measures ANOVA comparing baseline to follow-up neurocognitive performance as 
TABle 2: Descriptive Statistics for Baseline and 6-Month follow-up of Cardiovascular Fitness, Neurocognitive Screen, Eating Behavior, Body Composition, and Blood Markers.

\begin{tabular}{|c|c|c|c|c|}
\hline & $\begin{array}{c}\text { Baseline } \\
\mathrm{M}(\mathrm{SD})\end{array}$ & $\begin{array}{l}\text { 6-Month } \\
\mathrm{M}(\mathrm{SD})\end{array}$ & $\mathrm{F}$ & $P$ \\
\hline Cardiovascular fitness & & & 7.86 & $0.009^{*}$ \\
\hline 2-minute step test & $75.56(17.97)$ & $85.11(17.58)$ & & \\
\hline Neurocognitive screen performance & & & 4.61 & $0.04^{*}$ \\
\hline Modified minimental state exam & $90.03(7.14)$ & $87.83(8.53)$ & & \\
\hline Self-report eating behavior & & & 4.61 & $0.02^{*}$ \\
\hline Eating patterns & $4.72(2.17)$ & $3.86(1.92)$ & 9.51 & $0.005^{* *}$ \\
\hline Appetite questionnaire & $29.28(2.70)$ & $29.45(2.71)$ & 0.22 & ns \\
\hline Body composition & & & 0.71 & ns \\
\hline Body mass index & $26.90(5.02)$ & $26.84(5.00)$ & & \\
\hline Waist-to-hip ratio & $0.86(0.09)$ & $0.87(1.00)$ & & \\
\hline Blood markers (pg/mL) & & & 0.87 & ns \\
\hline Ghrelin & $16.633(9.01)$ & $15.63(8.60)$ & & \\
\hline Leptin & $17.96(13.68)$ & $17.11(12.30)$ & & \\
\hline Brain-derived neurotrophic factor & $17.37(9.01)$ & $16.10(8.39)$ & & \\
\hline
\end{tabular}

* Indicates significance at $P<0.05$ level; ** indicates significance at the $P<0.01$ level.

measured by the $3 \mathrm{MS}$ showed reduced overall performance $[\lambda=.86, \mathrm{~F}(1,29)=4.61, P=0.04]$.

\section{Discussion}

The current study examined the baseline relationships among variables associated with eating and weight maintenance, including physical fitness, eating behavior, neurocognitive function, body composition, and biomarkers in a sample of persons with MCI. In addition, the impact of a structured six-month program of aerobic and resistance training exercise on these variables was analyzed. Significant baseline relationships emerged among appetite and both cardiovascular fitness and neurocognition, as well as BMI and leptin. Following the exercise program, improvements in cardiovascular fitness and healthy eating habits occurred, while body composition indices and circulating biomarkers (leptin, ghrelin, and BDNF) remained stable. In contrast, decreased global cognition was observed following the exercise program.

As hypothesized, increased appetite was associated with a higher level of initial cardiovascular fitness and better baseline performance on a screening test of global cognition in our MCI sample. The positive association between appetite and fitness is consistent with research suggesting that physical activity increases food intake [11] and encourages further examination of whether a structured exercise intervention may promote appetite and other possible positive health outcomes in this population. In addition, the relationship between appetite and global neurocognitive performance indicated that appetite loss was associated with more severe cognitive impairment. This pattern is also consistent with past research indicating poor nutritional status and appetite in $\mathrm{AD}$ [7] and implicates the neuropathological changes associated with MCI as an important contributor to appetite loss. Thus, the beneficial effects of exercise on appetite may diminish over time in this population, underscoring the importance of early intervention.

Analyses examining the change from baseline to completion of the 6-month exercise program demonstrated the effectiveness of structured exercise in facilitating physical fitness and improving eating patterns, as significant improvement was observed in cardiovascular fitness and healthy eating habits from beginning to end. While appetite did not change significantly following completion of the exercise program, eating patterns did become healthier, and BMI and WHR remained stable. The reason for lack of appetite change is not entirely clear, but may be a product of this particular sample. Baseline appetite was within healthy ranges (i.e., above the cut-off indicating risk of weight loss) in all participants, suggesting a possible ceiling effect. Taken together, improved fitness, healthier eating patterns, and stable body composition indices suggest that the increased physical activity promoted healthy eating in this sample, while allowing weight to remain stable. Thus, increasing physical fitness through an exercise intervention may be of benefit in establishing healthy eating habits and stabilization of body mass in persons with MCI.

Despite improved cardiovascular fitness, neurocognitive screening performance declined in this group. Although differing from what has been found in cognitively healthy older adults [12-14], this result is consistent with what many have been observed in individuals who are already demonstrating degenerative impairment (e.g., nursing home participants) $[42,43]$. While exercise may be of cognitive benefit in the healthy aging process, the exercise program utilized in the current study did not appear to improve cognition in individuals for whom onset of pathological cognitive impairment had already begun.

Further, baseline to follow-up comparison of circulating biomarkers (leptin, ghrelin, and BDNF) showed no significant change. Given that previous research suggests exercise 
decreases ghrelin [24] while increasing BDNF [13] and leptin is associated with eating behavior [21], it was anticipated that the exercise program might decrease both ghrelin and leptin, while increasing BDNF. As cardiovascular fitness and eating behavior improved in the group, the stability of these blood markers is somewhat unexpected. It is noted that the current analyses did not control for possible effects of medical history or pharmacologic treatment on these biomarkers, and future work should investigate the possible contributions of such factors. However, as previously noted, these biomarkers have all been linked with neurocognitive processes. In light of the nature of the sample (i.e., persons with $\mathrm{MCI}$ ), as well as the evidence for overall cognitive decline in the group, it may be that the positive impact of the exercise program could not overcome the already declining cognition in this group.

Replication of these findings is necessary, as the current study is not without limitations. The sample of the current study was small, somewhat homogenous in premorbid characteristics (i.e., high level of education, highly active group), and heterogeneous is cognitive profile (i.e., including both amnestic and nonamnestic MCI, suggesting multiple etiologies of cognitive impairment, including cerebrovascular disease as well as $\mathrm{AD}$ ); as such, findings may not be entirely generalizable to other samples. Another limitation of the current study is that it was an exploratory study lacking a control group; this type of intervention should optimally be compared between individuals with MCI and other groups, including sedentary individuals with MCI and healthy older adults participating in an exercise program. Future directions include expanded sample size, variations in exercise (e.g., more intense or more frequent exercise), greater detail regarding participant previous dietary and exercise history, pharmacologic, medical, and family histories, genetic testing, and neuroimaging. In addition, future studies should also examine whether more objective measures of appetite (e.g., detailed caloric intake assessment) produce differential findings.

Taken together with previous research, results of this study suggest that although exercise is beneficial for cognition and cognitive biomarkers in the healthy aging process, it may not benefit individuals for whom onset of pathological cognitive impairment has already begun. Regardless, increasing physical fitness through an exercise intervention may be of benefit to this population in its association with healthier eating habits and stabilization of body compositions.

\section{Acknowledgments}

Data collection is supported by the Extendicare Foundation. This paper is supported in part by DK075119 and HL089311.

\section{References}

[1] J. A. Luchsinger and D. R. Gustafson, "Adiposity and Alzheimer's disease," Current Opinion in Clinical Nutrition and Metabolic Care, vol. 12, no. 1, pp. 15-21, 2009.

[2] J. I. Wallace, R. S. Schwartz, A. Z. LaCroix, R. F. Uhlmann, and R. A. Pearlman, "Involuntary weight loss in older outpatients: incidence and clinical significance," Journal of the American Geriatrics Society, vol. 43, no. 4, pp. 329-337, 1995.

[3] S. Guyonnet, F. Nourhashemi, S. Andrieu et al., "A prospective study of changes in the nutritional status of Alzheimer's patients," Archives of Gerontology and Geriatrics, vol. 26, no. 1, pp. 255-262, 1998.

[4] J. M. Burns, D. K. Johnson, A. Watts, R. H. Swerdlow, and W. M. Brooks, "Reduced lean mass in early Alzheimer disease and its association with brain atrophy," Archives of Neurology, vol. 67, no. 4, pp. 428-433, 2010.

[5] T. F. Hughes, A. R. Borenstein, E. Schofield, Y. Wu, and E. B. Larson, "Association between late-life body mass index and dementia: the Kame Project," Neurology, vol. 72, no. 20, pp. 1741-1746, 2009.

[6] B. Shatenstein, M. J. Kergoat, and I. Reid, "Poor nutrient intakes during 1-year follow-up with community-dwelling older adults with early-stage Alzheimer dementia compared to cognitively intact matched controls," Journal of the American Dietetic Association, vol. 107, no. 12, pp. 2091-2099, 2007.

[7] P. N. Wang, C. L. Yang, K. N. Lin, W. T. Chen, L. C. Chwang, and H. C. Liu, "Weight loss, nutritional status and physical activity in patients with Alzheimer's disease. A controlled study," Journal of Neurology, vol. 251, no. 3, pp. 314-320, 2004.

[8] Y. Gu, J. W. Nieves, Y. Stern, J. A. Luchsinger, and N. Scarmeas, "Food combination and Alzheimer disease risk: a protective diet," Archives of Neurology, vol. 67, no. 6, pp. 699-706, 2010.

[9] K. L. Smith and C. E. Greenwood, "Weight loss and nutritional considerations in Alzheimer disease," Journal of Nutrition for the Elderly, vol. 27, no. 3-4, pp. 381-403, 2008.

[10] J. E. Blundell, R. J. Stubbs, D. A. Hughes, S. Whybrow, and N. A. King, "Cross talk between physical activity and appetite control: does physical activity stimulate appetite?" Proceedings of the Nutrition Society, vol. 62, no. 3, pp. 651-661, 2003.

[11] S. Whybrow, D. A. Hughes, P. Ritz et al., "The effect of an incremental increase in exercise on appetite, eating behaviour and energy balance in lean men and women feeding ad libitum," British Journal of Nutrition, vol. 100, no. 5, pp. 11091115, 2008.

[12] S. J. Colcombe, K. I. Erickson, N. Raz et al., "Aerobic fitness reduces brain tissue loss in aging humans," Journals of Gerontology: Series A, vol. 58, no. 2, pp. 176-180, 2003.

[13] S. Colcombe and A. F. Kramer, "Fitness effects on the cognitive function of older adults: a meta-analytic study," Psychological Science, vol. 14, no. 2, pp. 125-130, 2003.

[14] S. J. Colcombe, K. I. Erickson, P. E. Scalf et al., "Aerobic exercise training increases brain volume in aging humans," Journals of Gerontology: Series A, vol. 61, no. 11, pp. 1166-1170, 2006.

[15] A. F. Kramer, S. J. Colcombe, E. McAulley, P. E. Scalf, and K. I. Erickson, "Fitness, aging and neurocognitive function," Neurobiology of Aging, vol. 26, supplement 26, pp. 124-127, 2005.

[16] N. T. Lautenschlager, K. L. Cox, L. Flicker et al., "Effect of physical activity on cognitive function in older adults at risk for Alzheimer's disease: a randomized trial," JAMA_Journal of the American Medical Association, vol. 300, no. 9, pp. 10271037, 2008.

[17] J. M. Burns, B. B. Cronk, H. S. Anderson et al., "Cardiorespiratory fitness and brain atrophy in early Alzheimer disease," Neurology, vol. 71, no. 3, pp. 210-216, 2008.

[18] Y. E. Geda, R. O. Roberts, D. S. Knopman et al., "Physical exercise, aging, and mild cognitive impairment a populationbased study," Archives of Neurology, vol. 67, no. 1, pp. 80-86, 2010. 
[19] T. J. Harris, C. G. Owen, C. R. Victor, R. Adams, and D. G. Cook, "What factors are associated with physical activity in older people, assessed objectively by accelerometry?" British Journal of Sports Medicine, vol. 43, no. 6, pp. 442-450, 2009.

[20] E. Jerlhag, E. Egecioglu, S. L. Dickson, M. Andersson, L. Svensson, and J. A. Engel, "Ghrelin stimulates locomotor activity and accumbal dopamine-overflow via central cholinergic systems in mice: implications for its involvement in brain reward," Addiction Biology, vol. 11, no. 1, pp. 45-54, 2006.

[21] I. S. Farooqi, E. Bullmore, J. Keogh, J. Gillard, S. O’Rahilly, and P. C. Fletcher, "Leptin regulates striatal regions and human eating behavior," Science, vol. 317, no. 5843, p. 1355, 2007.

[22] J. Gunstad, P. Schofield, R. H. Paul et al., "BDNF Val66Met polymorphism is associated with body mass index in healthy adults," Neuropsychobiology, vol. 53, no. 3, pp. 153-156, 2006.

[23] R. V. Considine, "Human leptin: an adipocyte hormone with weight-regulatory and endocrine functions," Seminars in Vascular Medicine, vol. 5, no. 1, pp. 15-24, 2005.

[24] J. Wang, C. Chen, and R. Y. Wang, "Influence of short- and long-term treadmill exercises on levels of ghrelin, obestatin and NPY in plasma and brain extraction of obese rats," Endocrine, vol. 33, no. 1, pp. 77-83, 2008.

[25] M. B. Spitznagel, A. Benitez, J. Updegraff et al., "Serum ghrelin is inversely associated with cognitive function in a sample of non-demented elderly," Psychiatry and Clinical Neurosciences, vol. 64, no. 6, pp. 608-611, 2010.

[26] J. Gunstad, M. B. Spitznagel, T. A. Keary et al., "Serum leptin levels are associated with cognitive function in older adults," Brain Research, vol. 1230, pp. 233-236, 2008.

[27] J. Gunstad, A. Benitez, J. Smith et al., "Serum brain-derived neurotrophic factor is associated with cognitive function in healthy older adults," Journal of Geriatric Psychiatry and Neurology, vol. 21, no. 3, pp. 166-170, 2008.

[28] S. Peng, J. Wuu, E. J. Mufson, and M. Fahnestock, "Precursor form of brain-derived neurotrophic factor and mature brainderived neurotrophic factor are decreased in the pre-clinical stages of Alzheimer's disease," Journal of Neurochemistry, vol. 93, no. 6, pp. 1412-1421, 2005.

[29] C. Laske, E. Stransky, T. Leyhe et al., "Stage-dependent BDNF serum concentrations in Alzheimer's disease," Journal of Neural Transmission, vol. 113, no. 9, pp. 1217-1224, 2006.

[30] N. Pannacciulli, D. S. N. Le, K. Chen, E. M. Reiman, and J. Krakoff, "Relationships between plasma leptin concentrations and human brain structure: a voxel-based morphometric study," Neuroscience Letters, vol. 412, no. 3, pp. 248-253, 2007.

[31] A. Schanze, U. Reulbach, M. Scheuchenzuber, M. Gröschl, J. Kornhuber, and T. Kraus, "Ghrelin and eating disturbances in psychiatric disorders," Neuropsychobiology, vol. 57, no. 3, pp. 126-130, 2008.

[32] D. Bataar, k. Patel, and D. D. Taub, "The effects of ghrelin on inflammation and the immune system," Molecular and Cellular Endocrinology, vol. 340, no. 1, pp. 44-58, 2011.

[33] A. Erol, "An integrated and unifying hypothesis for the metabolic basis of sporadic Alzheimer's disease," Journal of Alzheimer's Disease, vol. 13, no. 3, pp. 241-253, 2008.

[34] K. S. Krabbe, E. L. Mortensen, K. Avlund et al., "Brain-derived neurotrophic factor predicts mortality risk in older women," Journal of the American Geriatrics Society, vol. 57, no. 8, pp. 1447-1452, 2009.

[35] J. C. Morris, "The Clinical Dementia Rating (CDR): current version and scoring rules," Neurology, vol. 43, no. 11, pp. 24122414, 1993.

[36] R. E. Rikli and C. J. Jones, Senior Fitness Test Manual, Human Kinetics, Champaign, Ill, USA, 2001.
[37] D. R. Thomas, W. Ashmen, J. E. Morley et al., "Nutritional management in long-term care: development of a clinical guideline," Journals of Gerontology: Series A, vol. 55, no. 12, pp. M725-734, 2000.

[38] A. S. Ammerman, P. S. Haines, R. F. DeVellis et al., "A brief dietary assessment to guide cholesterol reduction in lowincome individuals: design and validation," Journal of the American Dietetic Association, vol. 91, no. 11, pp. 1385-1390, 1991.

[39] M. M. G. Wilson, D. R. Thomas, L. Z. Rubenstein et al., "Appetite assessment: simple appetite questionnaire predicts weight loss in community-dwelling adults and nursing home residents," American Journal of Clinical Nutrition, vol. 82, no. 5, pp. 1074-1081, 2005.

[40] E. L. Teng and H. C. Chui, "The Modified Mini-Mental State (MMS) examination," Journal of Clinical Psychiatry, vol. 48, no. 8, pp. 314-318, 1987.

[41] R. C. Bland and S. C. Newman, "Mild dementia or cognitive impairment: the Modified Mini-Mental State Examination (3MS) as a screen for dementia," Canadian Journal of Psychiatry, vol. 46, no. 6, pp. 506-510, 2001.

[42] C. D. Mulrow, M. B. Gerety, D. Kanten et al., "A randomized trial of physical rehabilitation for very frail nursing home residents," Journal of the American Medical Association, vol. 271, no. 7, pp. 519-524, 1994.

[43] J. F. Schnelle, P. G. MacRae, K. Giacobassi, H. S. H. MacRae, S. F. Simmons, and J. G. Ouslander, "Exercise with physically restrained nursing home residents: maximizing benefits of restraint reduction," Journal of the American Geriatrics Society, vol. 44, no. 5, pp. 507-512, 1996. 


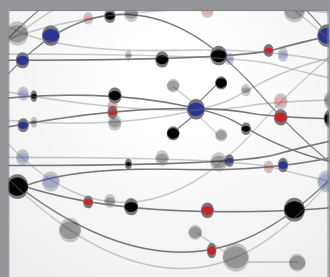

The Scientific World Journal
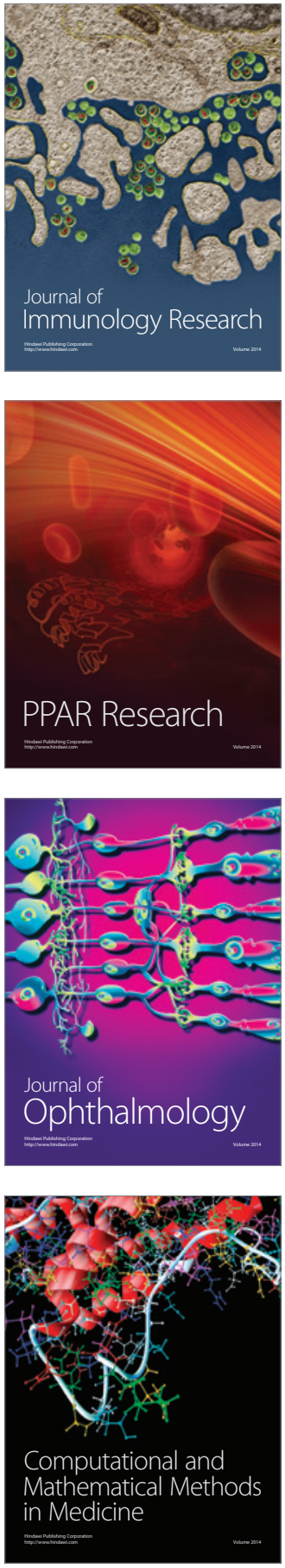

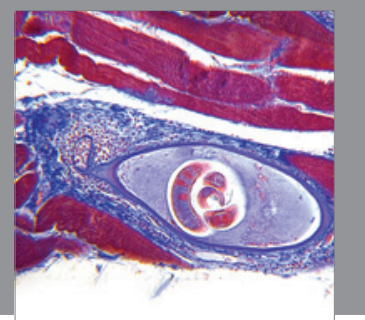

Gastroenterology

Research and Practice
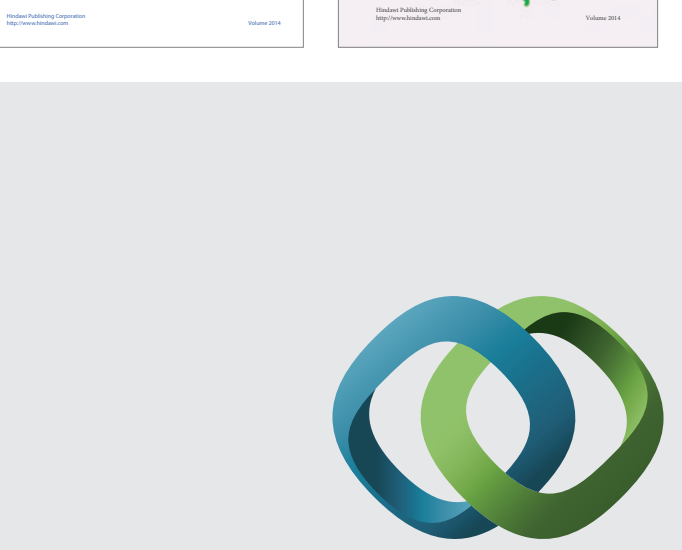

\section{Hindawi}

Submit your manuscripts at

http://www.hindawi.com
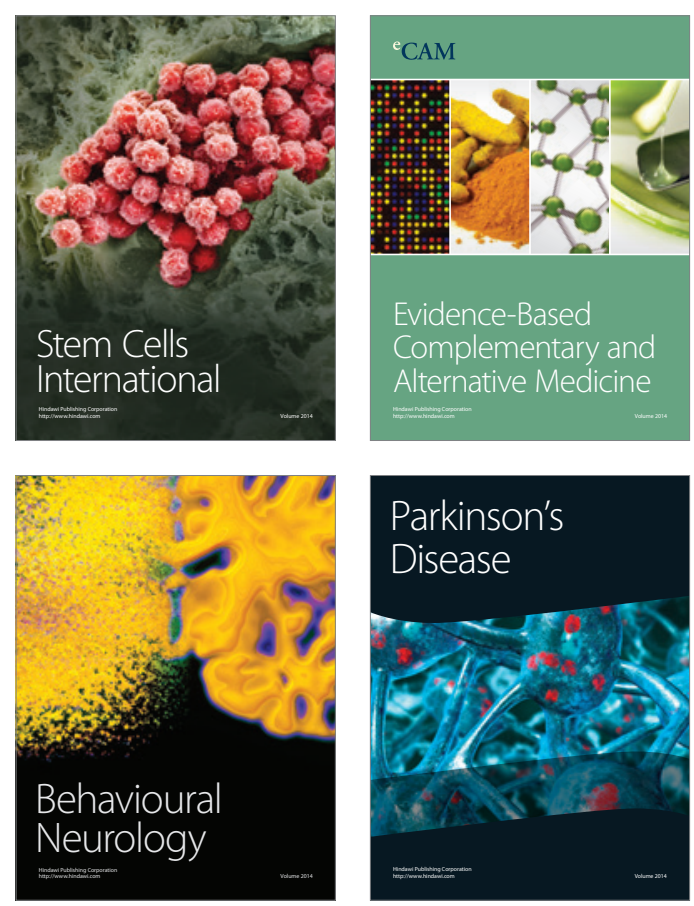

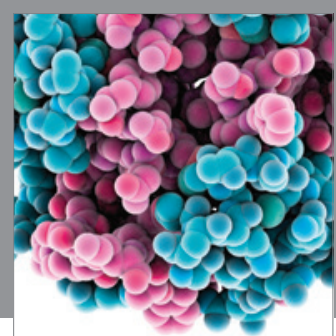

Journal of
Diabetes Research

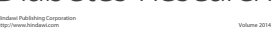

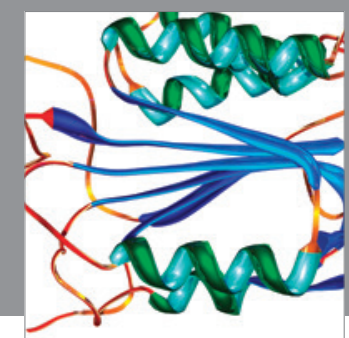

Disease Markers
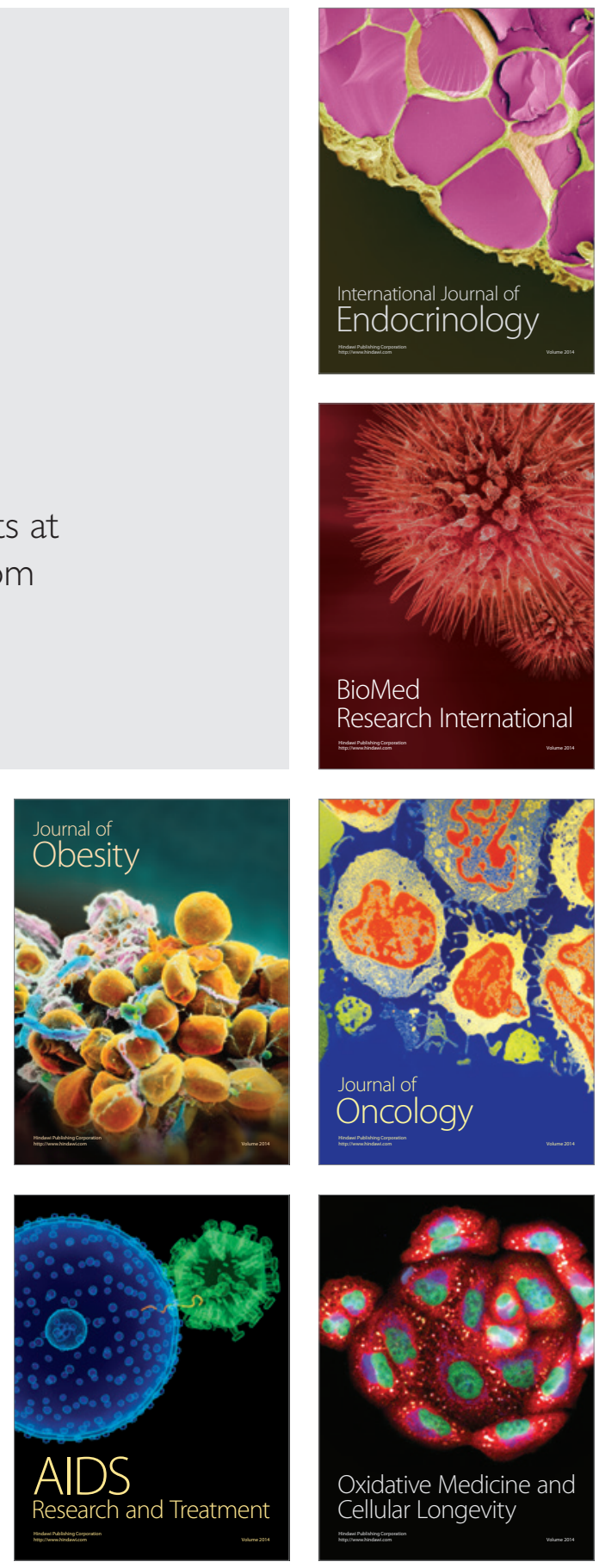